\title{
Surface Composition of Vesta: Issues and Integrated Approach
}

\author{
Carle M. Pieters • Lucy A. McFadden - Thomas Prettyman • M. Cristina De Sanctis • \\ Thomas B. McCord · Takahiro Hiroi · Rachel Klima · Jian-Yang Li · Ralf Jaumann
}

Received: 9 April 2011 / Accepted: 6 July 2011 / Published online: 27 August 2011

(C) The Author(s) 2011. This article is published with open access at Springerlink.com

\begin{abstract}
The instruments on the Dawn spacecraft are exceptionally well suited to characterize and map the surface composition of Vesta in an integrated manner. These include a framing camera with multispectral capabilities, a high spectral resolution near-infrared imaging spectrometer, and a gamma-ray and neutron spectrometer. Three examples of issues addressed at Vesta are: (1) What is the composition of Vesta's interior and differentiation state as exposed by the Great South Crater? (2) How has space weathering affected Vesta, both globally and at a local scale? and (3) Are volatiles or hydrated material present on Vesta's surface? We predict that Dawn finds many surprises, such as an olivine-bearing mantle exposed near the south-pole, a weakly or un-weathered surface that has been rela-
\end{abstract}

C.M. Pieters $(\varangle) \cdot$ T. Hiroi

Department of Geological Sciences, Brown University, Providence, RI 02912, USA

e-mail: Carle_Pieters@brown.edu

L.A. McFadden

NASA Goddard Space Flight Center, Greenbelt, MD 20771, USA

T. Prettyman

Planetary Science Institute, Tucson, AZ 87519, USA

M.C. De Sanctis

INAF, Istituto di Astrofisica Spaziale e Fisica Cosmica, Area di Ricerca di Tor Vergata, Via del Fosso del Cavaliere, 00133 Rome, Italy

T.B. McCord

Bear Fight Institute, 22 Fiddler's Road, Winthrop, WA 98862, USA

R. Klima

Applied Physics Laboratory, Johns Hopkins University, Laurel, MD 20723, USA

J.-Y. Li

Department of Astronomy, University of Maryland, College Park, MD 20742, USA

R. Jaumann

Institute of Planetary Research, Deutsches Zentrum fuer Luft- und Raumfahrt, Berlin, Germany 
tively recently resurfaced, and a very thin layer of surficial volatiles derived from interaction with the solar wind.

Keywords Vesta composition · Dawn mission · Great South Crater · Space weathering · Volatiles on asteroids

\section{Introduction}

Vesta is unique among main-belt asteroids. It is large, second only to Ceres in mass. Its surface is basaltic, implying that it has experienced a planetary scale differentiation that produced a crust and mantle and possibly a core. We have in our meteorite collection a suite of samples, the howardites, eucrites, and diogenites (HEDs; see McSween et al. 2010, this issue), most of which we believe came from Vesta. These samples, in turn, indicate that the formation of Vesta's crust and mantle occurred in the first 30 My of solar system history, well before larger terrestrial planets formed (see Coradini et al. 2011, this issue). Vesta may thus represent the type of proto-planet that was heated and differentiated early in solar system evolution and was subsequently accreted into the terrestrial planets (see Zuber et al. 2011, this issue).

Prior to Dawn's arrival at Vesta in 2011, all our information about this intriguing planetary body was derived from telescopic measurements and inferred from the HED meteorites, laboratory analogues, and theoretical modeling. A general review of telescopic measurements of Vesta and its relation to HED meteorites can be found in Pieters et al. (2006). As illustrated in Fig. 1, the spatial resolution enabled by HST has clearly shown there to be both albedo and color variations across the surface (e.g. Binzel et al. 1997; Thomas et al. 1997; Li et al. 2010). Rather than updating the overview of Vesta with more data and projecting what will be accomplished and discovered when Dawn arrives, we have selected three issues of scientific interest concerning the surface composition of Vesta that are widely discussed within the science community. We highlight these issues as case-study examples to illustrate how the suite of instruments on Dawn can be used in an integrated manner to address fundamental science at Vesta. The three themes discussed below are presented as examples of fundamental science issues that benefit from the integrated approach enabled by Dawn's complementary instruments (Russell et al. 2007).

Dawn's instrument suite was designed to characterize several aspects of Vesta's surface composition. The range of spatial information obtained by each instrument provides essential geologic context needed to understand processes that occurred during early surface evolution. Table 1 summarizes the characteristics of the three Dawn instruments and their fields of view are compared in Fig. 2. See Russell and Raymond (2011, this issue) for an overview of the mission operational timeline.

The Framing Camera $(F C)$ system consists of two identical cameras that will create global maps of topography, albedo, and color using one broadband clear filter and seven band-pass filters across the extended visual wavelength range from 400 to $1000 \mathrm{~nm}$ (Sierks et al. 2010, this issue). Of all the Dawn instruments, the FC provides the highest spatial resolution information for the surface of Vesta. The scientific objectives for FC imaging contribute to the determination of the asteroids' origin and evolution by measuring the asteroids' shape and topography, mapping the surface geomorphology, and characterizing the geological context of surface features and properties. While photogeology provides the qualitative interpretation of two-dimensional images, the third dimension is needed for quantitative geological analyses in terms of surface slopes, volumes, and mass balance of materials. 


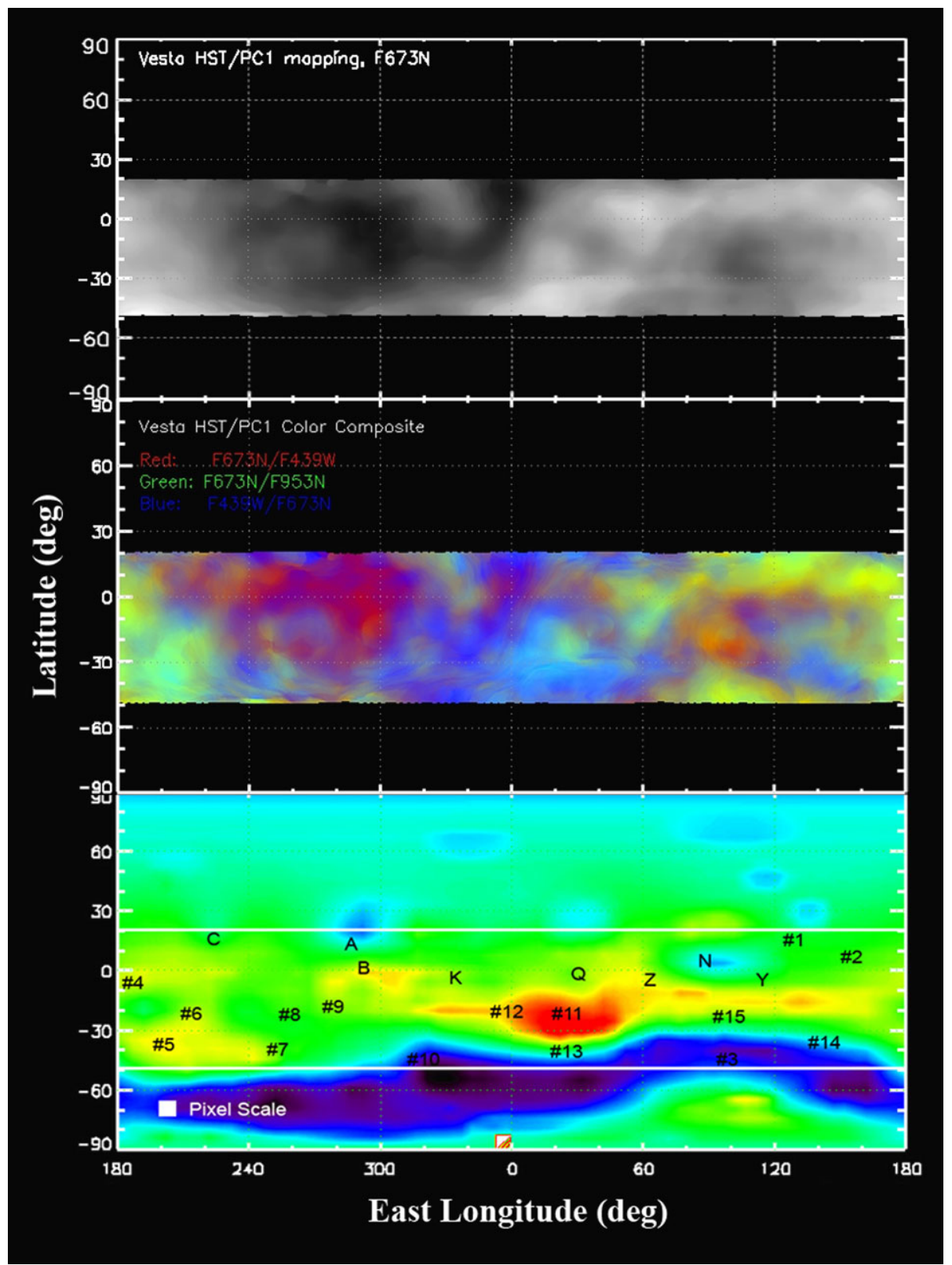

Fig. 1 Hubble Space Telescope measurements of Vesta (after Li et al. 2010). Top: Albedo of central latitudes at $673 \mathrm{~nm}$. Middle: Color composite of central latitudes similar to the standard Clementine color composite $\mathrm{R}=673 / 439, \mathrm{G}=673 / 953, \mathrm{~B}=439 / 673 \mathrm{~nm}$. Red-blue tones capture the visible continuum and green tones the relative strength of the $1 \mu \mathrm{m}$ absorption band. Bottom: Shape model of Thomas et al. (1997) with areas discussed by Li et al. (2010) identified 
Table 1 General properties of Dawn instruments for compositional analyses

\begin{tabular}{|c|c|c|}
\hline Instrument & Measurements & Data and Products \\
\hline $\begin{array}{l}\text { Framing Camera }(F C) \\
\text { (Sierks et al. } 2010 \text {, this issue) }\end{array}$ & $\begin{array}{l}\text { Broadband }+7 \text { spectral bands cen- } \\
\text { tered from } 438-965 \mathrm{~nm} \\
\text { FOV: } 5.5^{\circ} \\
\text { IFOV: } 93 \mu \mathrm{rad} \\
1024 \text { cross-track elements }\end{array}$ & $\begin{array}{l}\text { Visible albedo, Stereo imaging, } \\
\text { visible continuum, color units }\end{array}$ \\
\hline $\begin{array}{l}\text { Visual and Infrared Mapping } \\
\text { Spectrometer (VIR) } \\
\text { (De Sanctis et al. 2011a, this is- } \\
\text { sue) }\end{array}$ & $\begin{array}{l}\text { Two channels: } 0.25-1.05 \mu \mathrm{m} \text { and } \\
1-5 \mu \mathrm{m} \text {; each with } \sim 400 \text { spectral } \\
\text { bands } \\
\text { FOV: } 3.6^{\circ} \\
\text { IFOV: } 250 \mu \mathrm{rad} \\
256 \text { cross-track elements }\end{array}$ & $\begin{array}{l}\text { Vis-near IR albedo, mineralogy, } \\
\text { visible and near IR continuum, } \\
\text { temperature }\end{array}$ \\
\hline $\begin{array}{l}\text { Gamma Ray and Neutron } \\
\text { Detector (GRaND) } \\
\text { (Prettyman et al. 2011, this is- } \\
\text { sue) }\end{array}$ & $\begin{array}{l}\text { Thermal, epithermal, fast neutrons; } \\
\text { Gamma-ray: } 0-10 \mathrm{MeV} \text { (low resolu- } \\
\text { tion); 0-3 MeV (high resolution) } \\
\text { FOV: within the horizon } \\
\text { FWHM } \sim 1.5 \times \text { Altitude }\end{array}$ & $\begin{array}{l}\mathrm{H}, \mathrm{Fe}, \mathrm{Mg}, \mathrm{Si}, \mathrm{K}, \mathrm{Th} \text { abundance, } \\
\text { thermal neutron absorption, av- } \\
\text { erage atomic mass }\end{array}$ \\
\hline
\end{tabular}

Fig. 2 Dawn instrument Field of View at Vesta. The FOV for the Framing Camera (FC) and Visible Infrared Spectrometer (VIR) are shown for each of the three operational altitudes. VIR normally works in pushbroom mode indicated with arrows. GRaND operation occurs at the lowest orbit, LAMO. The image of Vesta includes the shape model of Thomas et al. (1997) derived from HST images

\section{DAWN Instrument Footprints}

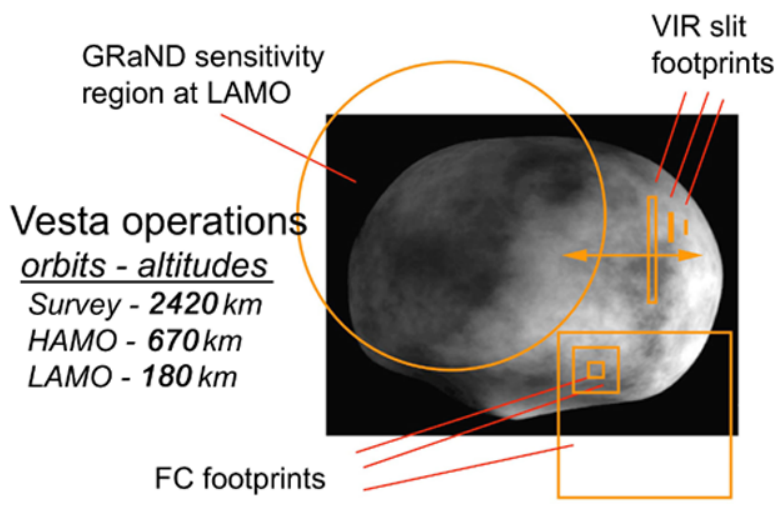

Detailed mapping of the topography is particularly important for understanding surface processes (Jaumann et al. 2007) and is accomplished at Vesta through extensive stereoscopic mapping (Raymond et al. 2011, this issue). FC multispectral imaging of the surface provides information on the spatial extent and character of compositional units. Age information of surface units is derived from crater frequency distributions measured from image mosaics. Relative ages, in context with the structural and compositional mapping, provide the stratigraphic sequence and thus are key to understanding the geologic history of the body.

The Visual and Infrared Mapping Spectrometer (VIR) experiment aboard the Dawn spacecraft is an advanced visible and infrared imaging spectrometer that operates from 0.25 to $5 \mu \mathrm{m}$ (De Sanctis et al. 2011a, this issue). The primary objectives of VIR mapping spectrometer at Vesta are the identification and characterization of different compositions and mixtures and the determination of their spatial distribution across the surface. Simultaneous high spectral- and spatial- resolution are needed to investigate surface geology, enabling the identification of mineralogical provinces, and production of 
maps of rock types and mineral composition. Such integrated maps provide information on the relationship between global and local spectral characteristics. Several diagnostic absorption bands for key minerals occur in the visible and near-infrared regions and can be identified with spectroscopic measurements (Burns 1993; Gaffey et al. 1993; Pieters and McFadden 1994). Common rock-forming minerals in both meteorites and asteroids exhibit distinctly different and diagnostic absorption bands. The wavelength, shape, and strength of various absorption features are determined by the minerals and molecules present within the first few millimeters of the surface. Each parameter must be measured accurately to make identifications and derive relative mineral abundances. The VIR mapping spectrometer has the resolution and accuracy necessary for this inversion process. VIS and Near IR spectroscopy contribute to asteroid studies by delineating absorption features which are not resolved with broad band filters and by refining the measurement of differences in spectral shape such as band depth and width. VIR will map the surface of Vesta with a spatial resolution of a few tens of meters, and will determine the spatial distribution of the various mineralogical types and their mixtures using both the diagnostic spectral features and the overall brightness.

The Gamma Ray and Neutron Detector $(G R a N D)$ is a segmented, deck-mounted nuclear spectrometer. A detailed description of the instrument, data reduction and analysis methods, and prospective results at Vesta can be found in Prettyman et al. (2011, this issue). The gamma ray spectrometer consists of a large-volume bismuth germinate (BGO) scintillator and an array of $\mathrm{CdZnTe}$ semiconductors. The spectrometer is sensitive to the range of gamma rays (300 $\mathrm{keV}$ to $10 \mathrm{MeV}$ ) produced by the decay of naturally-occurring radioisotopes (K, Th, and $\mathrm{U}$ ) and nuclear reactions induced by secondary particles (mainly neutrons) from cosmic ray interactions with the bulk surface. The neutron spectrometer, consisting of loaded scintillators (boron-loaded plastic and lithiated glass), is sensitive to leakage neutrons in the thermal, epithermal, and fast energy ranges. Neutron spectroscopy will measure the bulk thermal neutron absorption cross section and average atomic mass, both of which provide linear constraints on elemental abundance. In addition, neutron spectroscopy will provide high sensitivity measurements of the abundance of H. Gamma ray spectroscopy, corrected using neutron measurements, provides absolute abundances and ratios of specific elemental constituents of silicate minerals, including $\mathrm{O}, \mathrm{Mg}, \mathrm{Al}, \mathrm{Si}, \mathrm{K}, \mathrm{Ca}, \mathrm{Ti}, \mathrm{Fe}, \mathrm{Ni}, \mathrm{U}$, and Th. If the HEDs are representative of Vesta, then GRaND will be most sensitive to Fe, Si, and Mg. GRaND is sensitive to the composition averaged over depths of about $100 \mathrm{~g} / \mathrm{cm}^{2}$ (10 s of decimetres). The instrument response is generally omnidirectional, and as a result, the field of view (FOV) is to the horizon at the orbital altitude. The full width at half maximum (FWHM) of the spatial response spans an arc length on the surface of about $1.5 \times$ the orbital altitude. In the $460 \mathrm{~km}$ low altitude mapping orbit, GRaND will be sensitive to the composition of the great south polar crater. In addition to whole body abundances and maps of elemental abundances, GRaND will constrain mixing ratios of HED end members and enable calculation of normative mineral abundances for comparison with the direct measurement of mineralogy from VIR.

\section{Three Case Study Science Themes at Vesta}

Our perspective of Vesta changed forever when an associated family of small asteroids with Vesta-like properties, commonly called Vestoids, was discovered by Binzel and Xu (1993). The location of recent measurements of small Vestoids relative to Vesta are shown in Fig. 3. Not long thereafter measurements made with the Hubble Space Telescope by Thomas et al. (1997) showed that an enormous crater-like depression occurs near Vesta's 


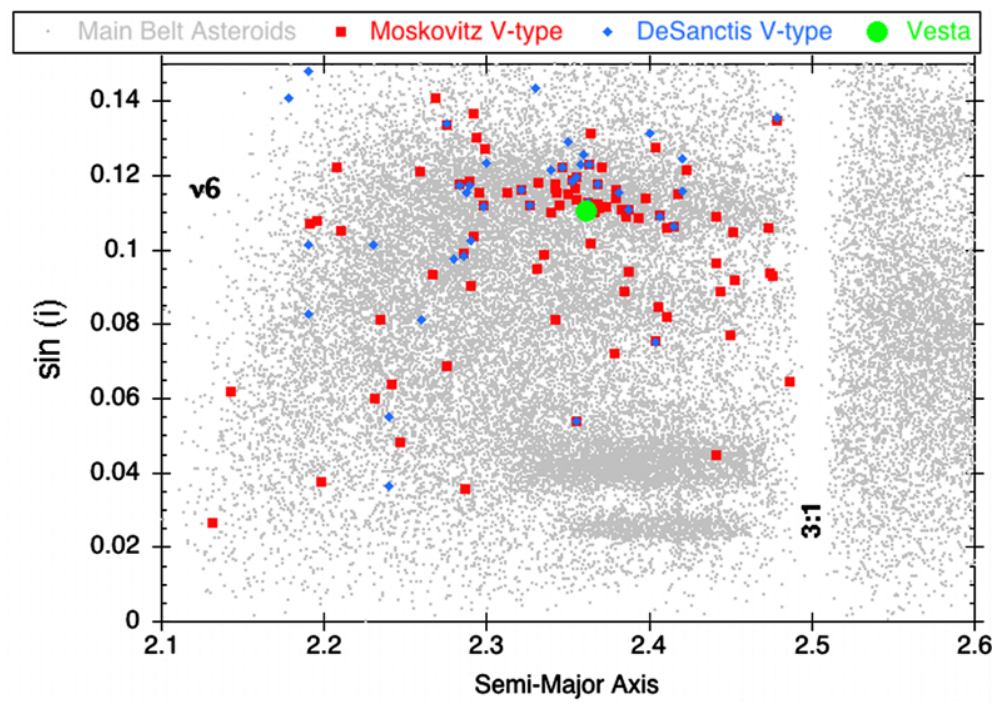

Fig. 3 Location of Vesta and the small asteroids with Vesta-like spectra as a function of inclination $(\sin (i))$ and Semi-major axis. Only the inner part of the main asteroid belt is shown, bounded by two prominent resonances. The main asteroid belt is characterized here by approximately 32 thousand main belt objects from the Sloan Digital Sky Survey (SDSS) catalogue (after Moskovitz et al. 2010, Fig. 1). All V-type asteroids identified by Moskovitz et al. (2010) and by De Sanctis et al. (2011b) are shown; several were independently studied by both groups. A few small V-type asteroids are identified beyond this range (not shown), only 5 of which are beyond the 3:1 resonances

south pole, the Great South Crater. What appears to be a central peak of this huge crater that dominates the southern hemisphere can be seen in Fig. 2. Debates have ensued about just when the formation of the Vestoids and the Great South Crater (GRSC) occurred in Vesta's history and whether or not they are related. The relative size of Vesta's GRSC provides a natural probe into the interior of this relatively small differentiated planetary body. This is a rare opportunity to directly compare the composition from the interior to the crust with the derived products from the mission's orbital phase. The interior of Vesta as exposed by the Great South Crater is thus one of the principal themes that can be uniquely addressed by Dawn's combined instruments. Our expectation derived from knowledge of the Earth's mantle composition and geophysical constraints is that Vesta's mantle is dominated by olivine. In the next section, we discuss various scenarios related to Vesta's crust and mantle composition and how they are addressed by Dawn's instruments.

Not long after the first spectroscopic measurements of Vesta were made that directly linked the asteroid to the basaltic achondrite family of meteorites using diagnostic mineral absorptions (McCord et al. 1970), Vesta became the center of an intense debate about if and how materials are altered in the space environment, or "space weathered" (e.g. see the range of early discussions in Chapman 1996). As illustrated in Fig. 4, the spectrum of Vesta contains diagnostic absorption bands that are almost identical to that of howardite meteorites (but only very fine-grained powders). Why did the surface of Vesta appear relatively pristine and similar to powdered achondritic meteorites whereas the soils of other airless silicate bodies like the Moon (and probably Mercury) have clearly experienced extensive space weathering and are quite different from powdered lo- 
Fig. 4 Telescopic spectrum of Vesta (circles) compared to laboratory spectra of different particle size separates for the howardite EET87503 (after Hiroi et al. 1994)

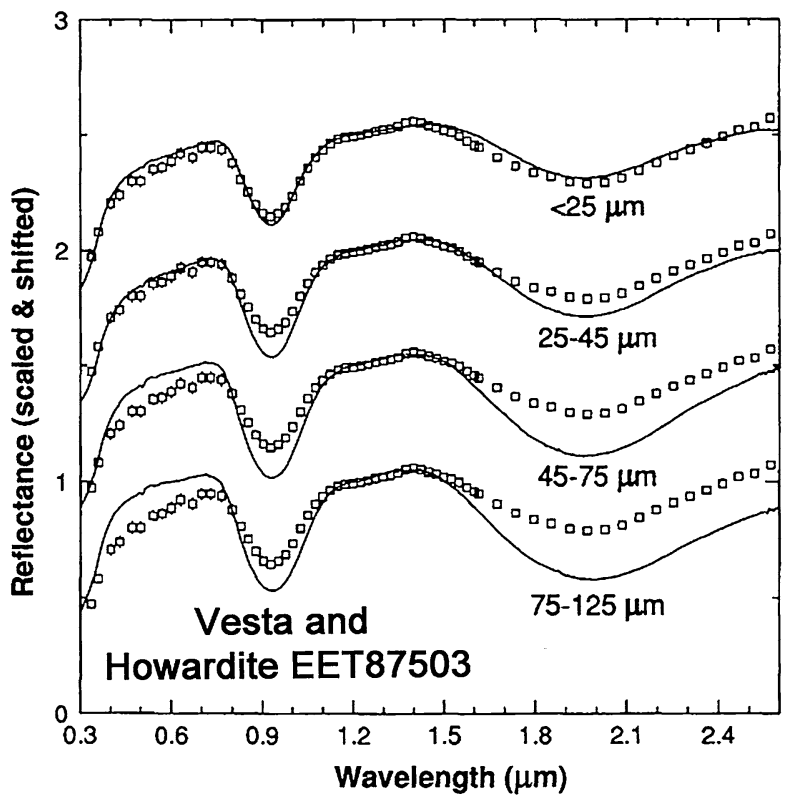

cal rocks? After decades of study, a principal cause (but not the precise origin) of the optical properties of lunar space weathering is now understood to be the development and accumulation of nanophase metallic iron, $\mathrm{npFe}^{0}$, on soil particles (Pieters et al. 2000; Hapke 2001). A similar accumulation of nanophase opaque particles appears to be operative to a lesser extent on some chondritic asteroid surfaces altering the surface to what is now observed as an abundant class of "S-type" asteroids. At the scale of telescopic measurements of large asteroids, however, Vesta remains unusual and exhibits minor if any weathering effects. For a still unknown reason or reasons, Vesta appears to be relatively immune to these widespread weathering processes. Only a few hints that surface soils may be altered have been detected. Thus, characterizing and identifying the extent and scale of any form of space weathering across Vesta's surface and at local features is vital to understand processes active on all airless bodies and is an ongoing quest and important theme for compositional study at Vesta.

The third case-study theme is the most recent. The study of extraterrestrial water has traditionally focused on comets, icy outer satellites Saturn's rings, and Mars. Anhydrous differentiated bodies like the Moon and Vesta were assumed to have lost any water early in their magmatic history and thus they were not relevant to understanding the role of water in the formation of the solar system. But how dry really is Vesta? The Moon has completely surprised us. The lunar interior is now believed to contain as much water as the Earth's depleted mantle (Saal et al. 2008; Hauri et al. 2011) and widespread surficial water and hydroxyl have been detected across the surface of the Moon (Pieters et al. 2009; Sunshine et al. 2009; Clark 2009). The instrument complement and measurement sequence at Vesta is well suited to identify the presence of any hydrous component and, if detected, to study its time and spatial variability. If water is found on Vesta the stage will be set for exploration of volatile processes on small airless silicate bodies. The possible presence of any form of water or hydrated material on Vesta is an intriguing and now compelling question. 


\section{Theme 1: Interior of Vesta as Exposed at the Great South Crater (GRSC)}

\subsection{GRSC: Background}

Although the HED meteorites provide partial constraints for the composition of Vesta, the structure of this proto-planet is unknown. Takeda $(1979,1997)$ proposed a layered-crust model of a parent body composed of howardite, eucrite and diogenite meteorites on the basis of x-ray diffraction and microprobe studies of pyroxenes in a suite of these basaltic achondrite meteorites. Observed exsolution and inversion textures reflect a crystallization trend controlled by the conditions on the parent body during formation. Their textures vary systematically with Mg and Ca concentrations. Miyamoto and Takeda (1994) estimated the burial depth of cumulate eucrites (Moore County) of about $8 \mathrm{~km}$, consistent with both the size of the Vestoid asteroids and the depth of the large crater in Vesta's southern hemisphere that Thomas et al. (1997) measure to be $13 \mathrm{~km}$. The Dawn mission's instrumentation reveal both chemical and mineralogical trends in pyroxenes from the VIR and GRaND instruments while the FC provides topography and albedo and color data along with more detailed geologic context.

Telescopic studies measuring the spectral reflectance of both Vesta and V-type asteroids associated with Vesta (Vestoids) have sought evidence of compositional variations across Vesta's surface and among the Vestoids (Gaffey 1997; Vernazza et al. 2005; Reddy et al. 2010, 2011; Li et al. 2010; Carry et al. 2010; Duffard et al. 2004; Moskovitz et al. 2010; De Sanctis et al. 2011b). Gaffey (1997) examined spectral variations as a function of longitude as Vesta rotates. He noted a particular region on Vesta where both albedo and spectral changes occur; the ferrous absorption band near $1 \mu \mathrm{m}$ is broader and occurs at a longer wavelength than the average pyroxene absorption band measured elsewhere and the albedo is higher than the average across the surface. These observations would be consistent with an olivine-rich region exposed at that longitude. Efforts to verify a possible olivine exposure have recently been carried out with additional earth-based telescopic measurements (Reddy et al. 2010) and by analyzing the colors of Vesta with Hubble Space Telescope (Li et al. 2010), but the spatial and spectral resolution was not sufficient to characterize the composition of Vesta's surface further.

Hubble Space Telescope imaging of Vesta revealed the very large crater in Vesta's southern hemisphere (Thomas et al. 1997), the Great South Crater (GRSC). The impact that created GRSC excavated into the interior and is expected to have removed or redistributed extensive material, perhaps including the Vestoids. Large craters such as the GRSC probe the interior and are thus a likely source of the range of observed achondrite HED meteorites (see McSween et al. 2010). The HEDs are delivered to Earth, directly from Vesta or indirectly from Vestoids, via chaotic motion such as proposed by Wisdom (1985) and/or the Yarkovsky effect (Bottke et al. 2006). The measured depth of the GRSC is $\sim 13 \mathrm{~km}$ (Thomas et al. 1997), suggesting that the combined suite of instruments on Dawn will look beneath the regolith deeper into the layers of the crust and/or mantle, including the cumulate eucrites studied by Miyamoto and Takeda.

The Dawn mission is well suited to map the GRSC in detail and use it as a probe to the interior of Vesta. The geometry of the orbital phase of the mission favors observing the southern hemisphere of Vesta (Russell and Raymond 2011). High spatial resolution is obtained by the framing camera (FC) providing detailed geologic context, high spectral resolution across the visible and infrared is obtained by VIR providing spectroscopy needed to discriminate pyroxene composition and detect the presence of olivine or other mineral constituents, and coarse-scale (inner-vs outer-basin) elemental abundances are measured by GRaND providing a direct link to solar system materials. 
Key questions related to Vesta's interior include:

1. Is a layered crust model of Vesta supported by observations of the walls, floor and central peak of the Great Southern Crater?

2. What compositional variations are observed with depth? Can specific stratigraphic layers be discerned? Are there parallel layers or is there evidence of a more complex evolution?

3. Are lateral variations in composition observed that are distinct from stratigraphic layering (which would suggest spatial heterogeneity of the crust and/or regional deposits)?

4. Is there evidence of diverse basaltic magmatism across the surface, implying varying composition of the interior source region in both space and time?

5. Alternatively, is there evidence for a conglomerate model where significant disruption during the crater forming event that produced the great southern crater resulted in a partial rubble pile or a reaccreted conglomerate?

\subsection{GRSC: Dawn Science Objectives}

Observations of the GRSC with the FC, VIR and GRaND contribute to assessing the character and heterogeneity of materials as exposed on the surface of Vesta and in the crater's interior. Note that there are multiple large craters on Vesta as evidenced by albedo maps. Our focus here is on the southern crater, though the concepts and anticipated results apply to all large and deep craters. Integrating results from all three instruments and including knowledge of the gravity field derived from analysis of the spacecraft's orbit will constrain hypotheses of the interior structure of Vesta proposed above.

One hypothesis to be tested at Vesta is that its mantle and lower crust is revealed in and around the GRSC. The questions outlined above are addressed by examining and contrasting materials seen in the central peaks, the walls, and floor of the crater and surrounding ejecta.

\subsection{GRSC: Data Integration Approach}

In the prime geologic mapping orbit, HAMO, the framing camera images surface features in multiple filters at a spatial resolution of $100 \mathrm{~m} / \mathrm{pixel}$ and $\mathrm{S} / \mathrm{N}>50$ (Sierks et al. 2010, this issue). In the lowest orbit, LAMO, the spatial resolution is $\sim 17 \mathrm{~m} / \mathrm{pixel}$, but coverage is less. Stereo imaging permits measurement of the depth of the crater, slopes, thickness of different layers observed in the crater wall, and the characteristics of the crater floor and central peak(s). Combining albedo, color and topography, geological maps are produced from which the history of the surface is inferred. These data allow searching for evidence of layering on the crater's walls. The crater bottom may be covered by fall back ejecta, melt ponds or a combination of both. If the GRSC is old, there will be a history of superimposed craters that constrain the impact age by crater frequency distribution on the floor and ejecta.

The Visible and Infrared Spectrometer yields spectral reflectance measurements between $0.3-5.0 \mu \mathrm{m}$ of different topographic regions with a spectral resolution of $\delta \lambda / \lambda=0.01-0.002$ (De Sanctis et al. 2011a, this issue). The presence of surface mafic minerals is determined from the position of absorption bands in the 1- and 2-micron regions. Combining the position, strength and width of the absorption bands, the chemical mineralogy is inferred by comparison with laboratory spectra and modeling their combination. Pyroxene, plagioclase and possibly olivine are known to be present. Volatile-bearing species (hydrates, carbonates, sulfates and phosphates) exhibit vibrational overtone absorptions in the near-IR and are also plausible constituents anticipated in small abundance or localized areas. Spectral imaging combined with measurements of the thickness of layers will result in determining whether or not the mantle is revealed. 


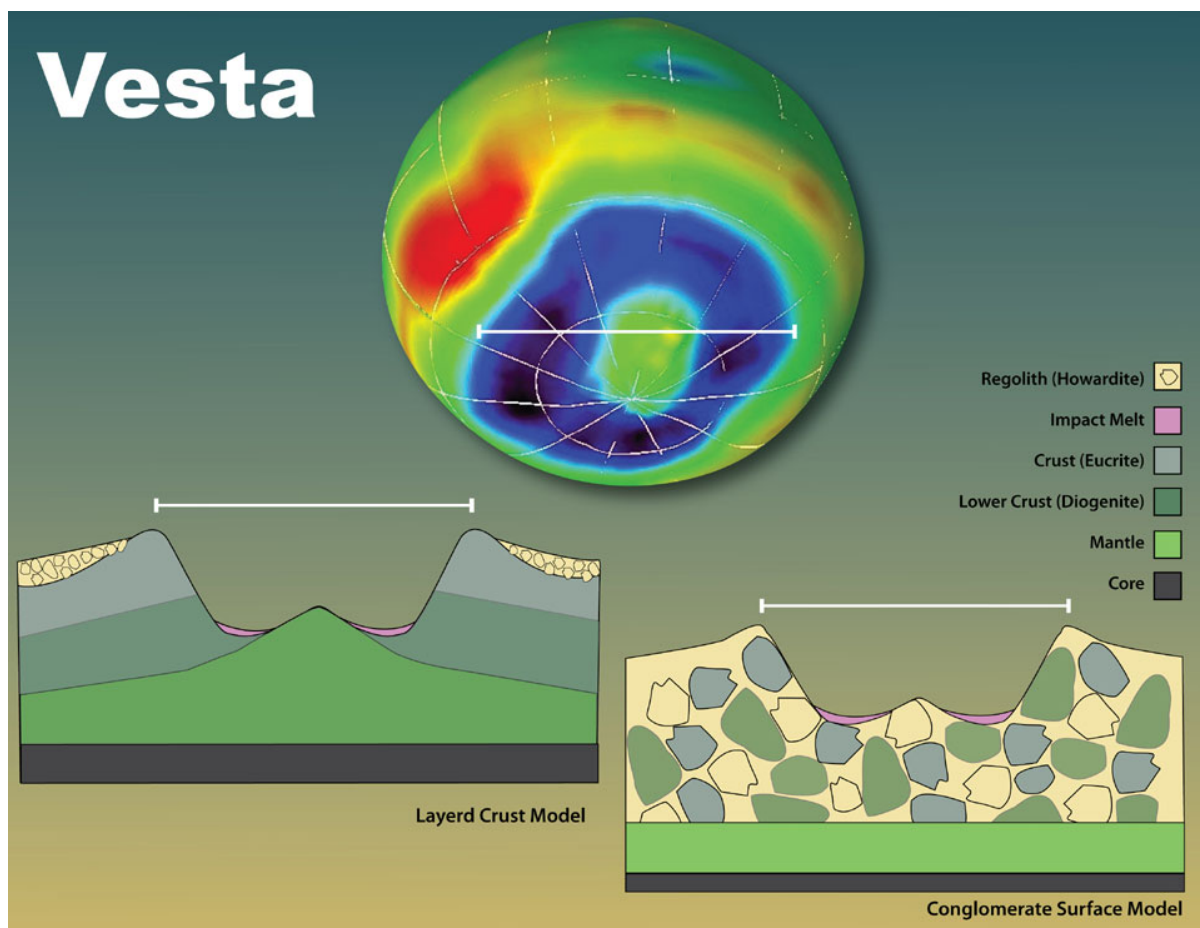

Fig. 5 Schematic cross section across Vesta's Great South Crater illustrating two possible scenarios for compositional diversity and structure of the crust

Prettyman et al. (2011, this issue) show that GRaND is sensitive to olivine-diogenite mixtures, which may be present in the GRSC. Such mixtures have a different neutron signature than whole rock HED abundances (lower absorption per average atomic mass). In addition, $\mathrm{Fe} / \mathrm{Si}$ and $\mathrm{Mg} / \mathrm{Si}$ ratios for olivine are measurably higher than for HEDs. These ratios can be determined by gamma ray spectroscopy. Scatterplots of quantities measured by GRaND would reveal the signature for the lower crust (ultramafic cumulates) and mantle (olivine) on the scale of the crater and possibly at higher resolution, depending on orbital altitude during measurements. GRaND data can be analyzed to determine elemental abundances on a smaller spatial scale, within the compositional units defined by VIR or FC, or by spatial deconvolution, providing information on the variation of elemental composition within the crater (e.g. diogenite-rich outer crater vs. an olivine-rich inner crater as illustrated by Prettyman et al. 2011).

\subsection{GRSC: Possible Outcomes}

We hypothesize that lower crust and possibly olivine-bearing mantle lithologies will be seen in the walls and central peak of the Great South Crater. Shown in Fig. 5 are two possible scenarios. In the first, Vesta's interior is layered as proposed by Takeda $(1979,1997)$ and the impact producing the Great South Crater exposes relatively pristine layers of Vesta's crust and its mantle, perhaps symmetrically about the impact point. With an energetic impact, the upper crust of Vesta would be excavated and deposited elsewhere, the relaxation of materials to the incident shock wave would produce a central peak, possibly revealing mantle material 
as a complex crater forms. Depending on how much regolith and/or impact melt accumulates in the bottom of the crater, components of the crust and mantle are revealed around the rim. On the other hand, simulation of an impact into a rotating Vesta (Jutzi and Asphaug 2011) predict a highly asymmetric, variably-shaped ejecta depositional sequence. Moreover, the simulations suggest that at the impact crater, material from as deep as $50 \mathrm{~km}$ is exposed and an inverse layering of the ejecta occurs next to the crater.

A second scenario has the event that produced the large crater also disrupting much of Vesta itself. In this scenario, there is reaccretion and reaggregation of large blocks the size of Vestoid asteroids, and the layered interior would be disrupted and jumbled. Compositional and elemental differences would be determined with either or both spectral and elemental mapping by VIR and GRaND. The distribution of material of a single composition would not necessarily be distributed symmetrically about the point of impact. The ability to determine this situation depends on the components of the conglomerate being distinguishable in reflectance and/or gamma-ray and neutron spectroscopy, as well as by units with color differences and morphology as seen from the framing camera. The distinguishing characteristic for this scenario is crater walls that show elemental and mineralogical variations that are not uniform in azimuth. Such results would be indicative of multiple crater/basin forming episodes throughout Vesta's history.

Experience with previous missions to the Moon and Mars suggests we can expect unusual and unexpected materials in the bottoms and sides of the large craters. These results will complement constraints from the gravity field measurements.

\section{Theme 2: Space Weathering on Vesta (Spwe)}

\subsection{Spwe: Background}

Space weathering (Spwe) refers to a plethora of processes that act on airless bodies in the harsh space environment. With time, exposed surfaces are often altered by processes that affect both the physical and compositional state in a manner that is dependent on the character of the surface (texture, starting composition, etc.) as well as solar system location (solar wind fluence, micrometeorite flux and energy, temperature, etc.). The effect of exposure is cumulative and thus not a simple story.

With the return of samples from the Moon by the Apollo and Luna missions it was quickly recognized that lunar soils, or regolith, have dramatically different physical and optical properties from those of broken or pulverized local rocks. Lunar soils were darker, absorption bands were greatly subdued, and the overall shape of the continuum was also altered. Decades later, when detailed analyses with modern equipment identified nanophase metallic iron $\left(\mathrm{npFe}^{0}\right)$ that had accumulated on the surface of lunar grains, it was recognized that these nanophase opaque particles are the principal cause of much of the observed optical properties of lunar soils (Keller and McKay 1993, 1997; Pieters et al. 2000; Taylor et al. 2001; Noble et al. 2001, 2007). Meteorite regolith breccias, as proxies for asteroidal regolith, were searched for similar nanophase particles, with the howardite Kapoeta being one of the few minor, but successful detections (Noble et al. 2010). Laboratory experiments have various degrees of success in simulating these particular space weathering products along with their optical effects (e.g., Cassidy and Hapke 1975; Moroz et al. 1996; Yamada et al. 1999; Sasaki et al. 2001; Hiroi and Sasaki 2001). A model describing the optical effects of nanophase metallic iron was successfully developed by Hapke (2001). The question remains: to what extent do the processes active 
on the Moon also occur on asteroids of the inner solar system and how are they manifested?

Reviews of evidence for space weathering on asteroid surfaces are given by Clark et al. (2002) and Chapman (2004). A recent perspective is also provided by Gaffey (2010). There has been a long-standing controversy over whether or not many S-type asteroids are the parent bodies of ordinary chondrites, but with a surface that has been altered in the space environment. The issue has been settled by gradual accumulating telescopic data for small asteroids with good spatial and spectral resolution (Binzel et al. 1996, 2004) as well as detailed compositional studies linking S-asteroids and ordinary chondrites during rendezvous of Eros by NEAR (McCoy et al. 2001; Cheng 2002; Bell et al. 2002) and asteroid 25143 Itokawa by Hyabusa (Binzel et al. 2001; Hiroi et al. 2006; Ishiguro et al. 2007). Reports on analyses of Itokawa material returned to Earth were presented at the Lunar and Planetary Science Conference in Houston (March, 2011) and showed that indeed Itokawa is composed of LL chondritic material with several particles exhibiting the presence of small amounts of a nanophase metal-rich component indicating spaceweathering. Although the details and rates remain to be explored, the concept of alteration in the space environment during regolith formation on meteorite parent bodies (specifically ordinary chondrite) as well as the Moon is generally well accepted.

\subsection{Spwe: Dawn Science Objectives}

Vesta nevertheless remains enigmatic. Since the discovery that Vesta's near-infrared reflectance spectrum is closely comparable to laboratory measurements of particulate samples of unweathered HED meteorites (McCord et al. 1970), it has been believed that Vesta's surface shows little or no evidence of space weathering alteration. On the other hand, laboratory experiments conducted on HEDs, and pyroxene constituents, suggest that Vesta-like materials should indeed alter under the effects of ion bombardment and micrometeorite impacts in a way similar to that observed for ordinary chondrite-like materials, but at Vesta the alteration timescale is longer (Hiroi and Pieters 1998; Marchi et al. 2005; Vernazza et al. 2006). The many controlling variables on the rate of changes with space weathering are poorly constrained. One evolving model for the rate of space weathering on asteroids using age estimates for family members of a few apparently recent asteroid breakups (Nesvorný and Vokrouhlický 2006) along with asteroid measured color suggest that color changes associated with space weathering may be very rapid $\left(<10^{6} \mathrm{yr}\right)$ (Vernazza et al. 2009). For Vesta, the measured crystallization ages of the HED meteorites, however, indicate that the original surface of Vesta is older than the lunar highlands (e.g., McSween et al., 2010). Thus, the surface of Vesta has had ample opportunity to develop a regolith comparable to other asteroids, unless special events have been encountered.

Under closer scrutiny, the spectrum of Vesta is not directly comparable to typical HED spectra in band strength. Although the prominent mineral absorption bands at 1 and $2 \mu \mathrm{m}$ of Vesta have the same shape and wavelength as howardites, they are shallow (weaker) when compared to reflectance spectra of typical HED meteorites measured in the laboratory. Assuming the bulk mineralogy of Vesta is not greatly different from the observed range seen in HED meteorites, this minor difference in band strength could simply indicate the asteroid surface has a distinctly fine-grained regolith globally (Hiroi et al. 1994) as illustrated in Fig. 4. An alternative explanation for the weaker bands observed for Vesta is that the surface has developed a regolith derived from plagioclase-containing HEDs and the finest particles of the regolith are enriched with this feldspathic component and dominate the measured properties. This feldspathic enrichment of the finest fraction has been well-documented for 
soils on the Moon (Pieters and Taylor 2003). However, an additional explanation is required to account for differences between Vesta and the family of small asteroids apparently associated with Vesta (the Vestoids of Fig. 3) that exhibit similar but stronger diagnostic mineral absorption bands. The strength of the bands observed for the Vestoids are much closer to that observed in laboratory studies of meteorites (Binzel and Xu 1993; Hiroi et al. 1995; Pieters et al. 2006). Marchi et al. (2010), Moskovitz et al. (2010), and De Sanctis et al. (2011b) investigated several of the small Vestoids using earth-based telescopes and found that their visible slopes are generally redder than that of Vesta and systematically redder than HEDs measured in the laboratory. The observed trend for Vestoids (steeper continuum, stronger absorption bands) is opposite that observed for S-type asteroids (steeper continuum, weaker absorption bands), again suggesting that Vesta and its family of small asteroids is distinctive.

Our currently poor understanding of the relative role of micro-meteorites, sputtering, solar wind irradiation, presence or absence of metallic iron or troilite, and the timing and abundance of large and small impacts onto Vesta has led to a diversity of predictions and hypotheses about the space weathering environment at Vesta and resulting regolith formation that would account for Vesta's prominent absorptions and relatively unweathered surface. For example, if solar wind protons are found to play a significant role in controlling space weathering products, the presence of an early core dynamo in Vesta could create magnetized surface materials that might shield all or part of the surface (Vernazza et al. 2006) perhaps resulting in reduced development of $\mathrm{nFe}^{0}$. Alternatively, the simplest explanation is that the materials on the surface have been recently exposed and generally have not had time to develop weathering products (Pieters and Binzel 1994; Wasson et al. 1996). Recent multispectral measurements across the surface of Vesta with HST have clearly demonstrated that there are nevertheless both albedo variations across the surface as well as color variations. When coupled to the shape model of Thomas et al. (1997), there are suggestions that optical properties and topography are coupled in several areas, suggesting regolith alteration and/or movement (Li et al. 2010).

Why Vesta's surface appears to be relatively unaltered and does not show significant space weathering is one of the key questions that Dawn addresses.

\subsection{Spwe: Dawn Data Integration/Approach}

All three primary instruments of Dawn contribute key information to understanding the space weathering environment and its products at Vesta. The FC provides first-order distinction by contrasting albedo and color of materials excavated at recent craters relative to surrounding older materials. If stratigraphic diversity is excluded based on geologic context and scale, then any distinctions between the crater and surroundings are due to accumulated environmental processes for the soils at Vesta, and this information sets a primary constraint on active regolith processes. Although FC color ratios can identify and map distinctions across the surface, they cannot characterize the mineralogy. Evaluation of the albedo and relative strength of the ferrous $1 \mu \mathrm{m}$ absorption band can nevertheless be linked with FC and VIR measurements for the same areas. VIR can then evaluate the full strength and character of the 1- and 2- $\mu \mathrm{m}$ diagnostic absorption bands as well as near-infrared continuum slope, both of which have been shown to be sensitive indicators of exposure for small craters on the Moon (Nettles et al. 2010). Laboratory experiments suggest that the composition of the surface also has an effect on the degree of space weathering products. For example, olivine is much more susceptible to space weathering than pyroxene (Hiroi and Sasaki 2001). An olivine-rich surface is predicted to exhibit more space weathering prod- 
ucts than a surface of comparable exposure age that is dominated by pyroxene. VIR is well suited to explore such issues in tandem with FC data for diverse regions. On the global scale, GRaND measures $\mathrm{H}$ abundance across the surface and this can be evaluated as a marker for total exposure to solar wind.

\subsection{Spwe: Possible Outcomes}

Two very different possible situations might be found at Vesta. (1) The surface is ancient and heavily cratered. In this case, if no apparent alteration is detected, e.g., morphologically fresh craters show no difference from background soils, then the environment at Vesta and/or its surface composition must be responsible. For example the lack of abundant metallic iron or troilite (FeS) might slow space weathering to unnoticeable levels. (2) Alternatively, large expanses of the surface are seen to have experienced a relatively recent resurfacing event. This implies there has been insufficient time to produce alteration products. This event could be linked to the formation of the Great South Crater or it could be an aftermath of numerous (but relatively global) smaller events.

Dawn data resolves this dilemma. Shown in Fig. 6 are schematic figures of what the surface of Vesta might look like for the situations described above. The top image is a heavily cratered surface that has resulted from up to $4.5 \mathrm{Ga}$ of cumulative impact bombardment. Old craters are degraded and superimposed, and all craters follow a regular size frequency distribution suggesting an old surface with a well developed regolith. Only a few small fresh craters are observed. The middle image depicts the same ancient surface, but the character of the surface has been affected by a large number of small more recent craters that have brightened and freshened the surface. The bottom figure depicts an old surface that has recently been disturbed by extensive deposits and mixing from a large-scale event, such as that which formed the Great South Crater. Small craters are preferentially removed and features morphologically associated with the resurfacing readily identified. This scenario is preferred-it is consistent with most of the available data and is the easiest to verify or negate (crater statistics, surface morphology, and spectroscopic relation between small craters and their surroundings).

\section{Theme 3: Possible H, Hydroxyl, Water and Other Volatiles on Vesta?}

\subsection{Water: Background}

Recent reports of water or at least excess hydrogen and other volatiles on/in the Moon's surface have changed how we think about the surfaces of inner Solar System airless silicate bodies, such as Vesta. Measurements by Lunar Prospector's neutron spectrometer revealed excess hydrogen in lunar polar areas where there are permanently shadowed regions; however, the chemical form of $\mathrm{H}$ could not be determined from measurements of neutrons (Feldman et al. 1998, 2000, 2001; Lawrence et al. 2006; Eke et al. 2009). Excess hydrogen at the poles was also detected by the Lunar Reconnaissance Orbiter (LRO) mission's Lunar Explorer Neutron Detector (LEND) (Mitrofanov et al. 2010a, 2010b, 2010c; Lawrence et al. 2010), confirming the earlier findings. The Moon Mineralogy Mapper $\left(\mathbf{M}^{3}\right)$ imaging spectrometer on Chandrayaan-1 recently reported the widespread occurrence of reflectance spectrum absorptions in the 3- $\mu \mathrm{m}$ spectral region that appear the same as is commonly seen for $\mathrm{OH}$ and $\mathrm{H}_{2} \mathrm{O}$-rich surfaces in the laboratory and other parts of the solar system (Pieters et al. 2009). This result was verified and elaborated on by Clark (2009) from reanalyzed 
Fig. 6 Three schematic scenarios for Vesta's poorly weathered surface (see text for implications). Top: Ancient heavily cratered surface. Middle: Ancient heavily cratered surface that has abundant recent small craters. Bottom: Ancient heavily cratered surface that has been largely resurfaced by a regional/global event
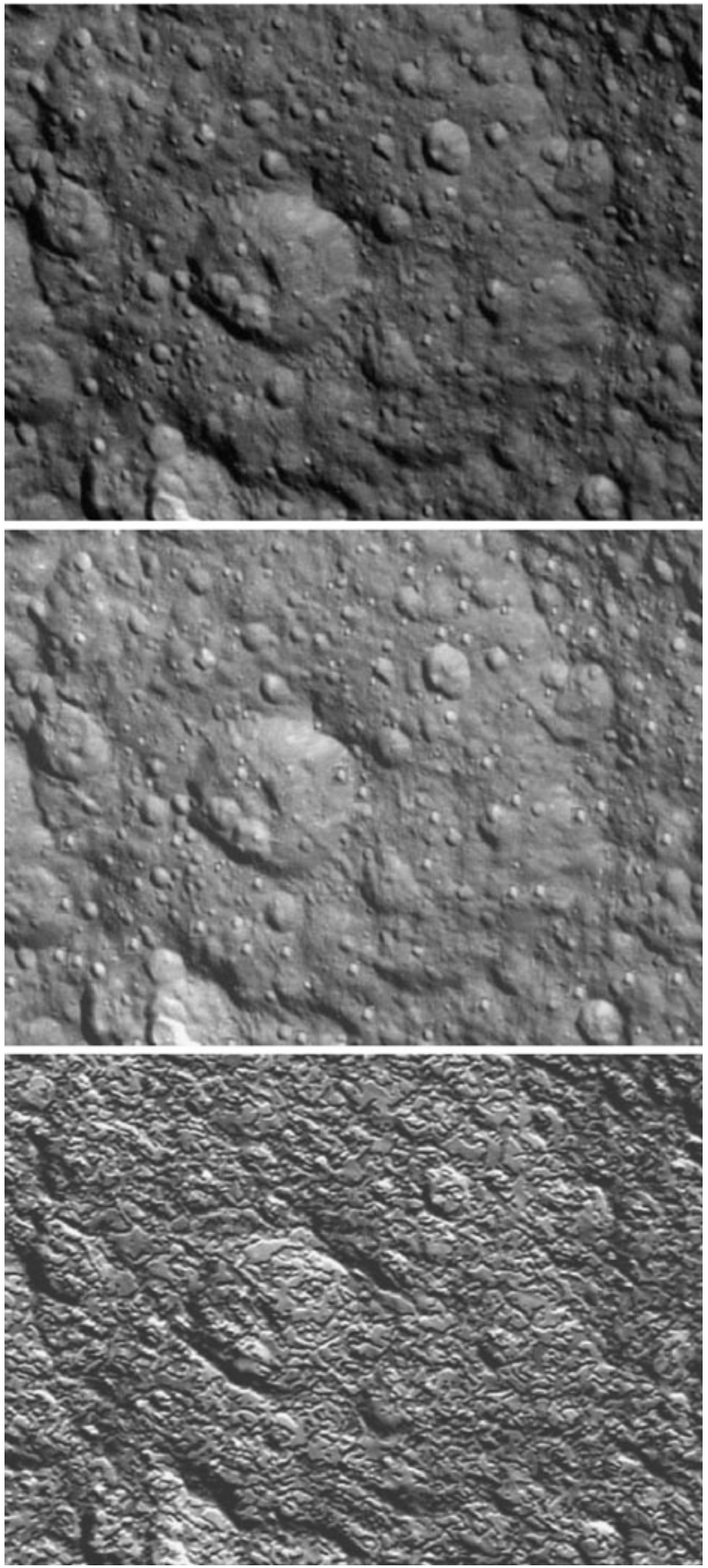

earlier Cassini VIMS spectroscopic observations, and by Sunshine et al. (2009) using new observations by the infrared spectrometer aboard the Deep Impact spacecraft. The Lunar Crater Observation and Sensing Satellite (LCROSS) experiment impacted the Centaur up- 
Fig. 7 Reflectance spectra of Vesta, a HED meteorite, and Mars. Mars and the meteorite spectra show $\mathrm{OH}$ absorptions near $3 \mu \mathrm{m}$. The Vesta spectrum exhibits strong ferrous bands near 1 and $2 \mu \mathrm{m}$, but has only weak or no $\mathrm{OH}$ absorption

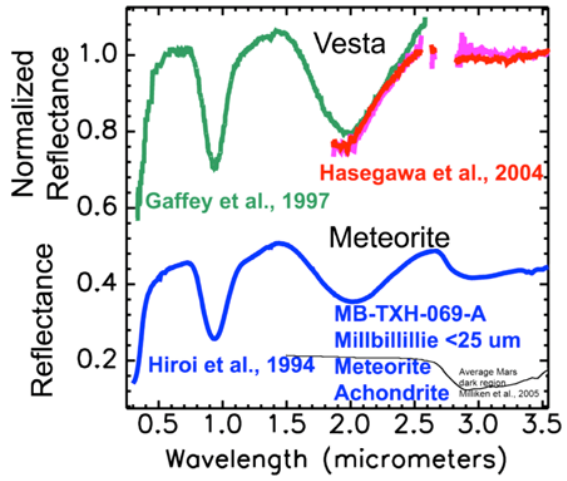

per stage rocket into a shadowed part of a lunar polar crater in October 2009, and evidence of water ice and other volatile materials were reported in the resulting excavated plume (Colaprete et al. 2010a, 2010b; Schultz et al. 2010).

Of particular interest is the presence of water and its constituents on other airless bodies in the solar system. $\mathrm{OH}$ and $\mathrm{H}_{2} \mathrm{O}$ on or in an airless object such as the Moon or Vesta can come from three major sources: (1) intrinsic to the material forming the planetary body, (2) from in-fall of cometary and hydrated meteorite materials, and (3) from solar-windproton-induced hydroxylation.

Solar wind induced hydroxylation is most likely of the available processes to be responsible for the $\mathrm{M}^{3}$ observations of widespread $\mathrm{OH} / \mathrm{H}_{2} \mathrm{O}$ (McCord et al. 2009, 2010a, 2010b, 2010c). This finding is based on the behavior of the $\mathrm{M}^{3} \mathrm{OH} / \mathrm{H}_{2} \mathrm{O}$ spectral features relative to Moon physical properties, on a considerable history of evidence of solar wind interactions with the surface material, and on a number of laboratory studies showing evidence of proton induced hydroxylation by irradiation (e.g. Zeller et al. 1966; Zent et al. 2010). Dawn is almost certainly able to detect and map any $\mathrm{OH} / \mathrm{H}_{2} \mathrm{O}$ in Vesta's surface layer due to this process, as it should be as sensitive as was $\mathrm{M}^{3}$ but with better spectral and temporal coverage.

Detailed petrographic analyses of the eucrite Serra de Magé identified the presence of veins that contain quartz likely deposited from liquid water solution (Treiman et al. 2004). It was hypothesized that these were short-lived events on the parent body Vesta, possibly derived from comet impacts. Telescopic observations of Vesta shown in Fig. 7 are said to hint at the presence, but currently show no strong evidence of water or $\mathrm{OH}$ on Vesta (Hasegawa et al. 2003; Rivkin et al. 2006). Vesta has an airless basaltic surface and is relatively warm and so would be expected to be very dry, as was the Moon. Still, some or all of the processes proposed to be responsible for the lunar surface water may also operate on Vesta. Differences in solar wind induced hydroxylation between the Moon and Vesta are likely, since Vesta is further out in the solar system and is cooler than the Moon. It is also possible that Vesta could accumulate more water/hydrate-rich material from the outer asteroid belt. On the other hand, if Vesta has a magnetic field, it might deflect the solar wind and inhibit $\mathrm{OH} / \mathrm{H}_{2} \mathrm{O}$ formation on the surface by the proton-induced hydroxylation.

Prettyman et al. (2011, this issue) point out that the average abundance of $\mathrm{H}$ in howardite, from chondritic clasts, is over four times higher than the lunar average $\mathrm{H}$, and suggest that the bulk (meter scale depth) H determined by GRaND at Vesta would provide constraints on the in-fall of hydrated meteoroids. Other volatiles might also be present. In general, ices are 
not thermally stable on or in the upper meters of the surface of Vesta, due to the relatively high temperatures, up to $\sim 250 \mathrm{~K}$, and lack of a substantial atmosphere. There could be venting from the interior from trapped deposits due to infall or from Vesta's evolution. $\mathrm{CO}_{2}$, for example, is a strong absorber in the IR spectrum and, if present, should be detected by VIR.

\subsection{Water: Dawn Science Objectives}

Detection of excess hydrogen, $\mathrm{OH}$ and $\mathrm{H}_{2} \mathrm{O}$, and other volatiles (e.g. $\mathrm{CO}_{2}$ ), are prime science objectives for Dawn, based on the recent experience with the Moon. These materials may be present in the surface, as they are on the Moon, due to several sources, and they may be relatively static deposits in the surface materials or due to active venting from the interior. The VIR measurements are of high value for detecting and mapping the several $\mathrm{OH}$ and $\mathrm{H}_{2} \mathrm{O}$ absorption features as well as other volatile that might be present. These molecules in general create strong absorptions in the infrared reflectance spectrum. GRaND measurements are also sensitive to the presence of hydrogen in any form, as a separate ion or molecule or incorporated in other molecules, such as $\mathrm{OH}$, but GRaND does not distinguish the molecular forms present. The VIR measurements of IR absorptions will be affected by contributions of radiation from thermal emission at longer wavelengths and require a careful assessment of temperature distributions and associated effects.

\subsection{Water: Dawn Data Integration Approach}

The two most important instruments on Dawn to study volatiles are VIR and GRaND, but they make very different measurements and have very different sensitivities. They each measure a different part of the surface material (VIR the top few millimeters and GRaND the top meter). GRaND is sensitive to hydrogen atoms but cannot distinguish among the molecular forms. VIR is sensitive only to the molecular forms but can distinguish several. The two instruments acquire data during different periods of Dawn activities at Vesta. The prime period of VIR data acquisition is during the Survey orbits early in the mission timeline when almost the entire surface will be acquired. More limited coverage is forseen during the high altitude orbit, HAMO, and low altitude orbit, LAMO, but with increasing spatial resolution. The prime period for GRaND data acquisition is later during the extended low altitude orbit period, LAMO, where GRaND integrates data from many orbits, building up a global map uniformly over time. As a result, VIR will be sensitive to time-varying events, depending on its spatial viewing, while GRaND will be sensitive to large-scale, time-integrated effects. When VIR and GRaND observations and results are both available, of particular interest are spatial and relative abundance comparisons between the detections of $\mathrm{H}$ and $\mathrm{OH} / \mathrm{H}_{2} \mathrm{O}$ molecules.

\subsection{Water: Possible Outcome}

Finding volatiles of any type on/in Vesta would be unexpected. Vesta is an airless body with a surface temperature high enough to prevent preserving most volatile over geologic time. The most likely sources of volatiles are from exogenic processes, such as solar wind implantation, or due to transient events, such as recent cometary impacts. Volatiles from 
either of these sources could migrate or be gardened into the surface regolith over time, and so the deposits could be secondary to the original source. Thus, any findings must be carefully analyzed for cause and effect.

The Vesta measurements provide a critical constraint on how $\mathrm{OH} / \mathrm{H}_{2} \mathrm{O}$ forms on airless bodies of the solar system, whether these molecules are found or not. The proposed source of these molecules is solar-wind proton implantation and combination with unsatisfied oxygen bonds on the soil grain surfaces and in internal crystal defects to form $\mathrm{OH}$ and $\mathrm{H}_{2} \mathrm{O}$. Finding $\mathrm{OH}$ on Vesta with properties consistent with this hypothesis would be a powerful confirmation, and it would support the proposal that this is a widespread process.

Not detecting $\mathrm{OH}$ may not be definitive. There are several possible explanations for nondetection. Certainly, the solar wind should still be a source of hydrogen at Vesta. Compared to the Moon's surface, the solar wind at Vesta should have about the same velocity and thus, per particle, about the same energy and penetration depth in the surface grains. But, the density of the wind at Vesta should be lower than at the Moon by a distancesquared relationship, or about a factor of 4.5, assuming the solar wind experiences approximately spherical expansion from the Sun. Thus, the same physical processes should be possible at Vesta as for the Moon, but perhaps operating at a slower pace by the above factor. However, the formation on the lunar surface seems to depend on the nature of the lunar soil particle, as well as on the properties of the solar wind. The grains are heavily physically damaged, due to micrometeorite impacts and radiation sputtering in a vacuum. This results in a very chemically reactive material with a high density of dangling oxygen bonds. Lunar soil grains have large surface areas and many internal crystal defects. Vesta's surface, on the other hand, seems to be relative more crystalline and undamaged (low space weathering) and therefore may be relatively chemically inactive. Also, $\mathrm{OH}$ formation on the Moon seems to be related to surface mineralogy and stronger for highly feldspathic material, which is less abundant on Vesta. So, although the solar wind should produce the same physical effect at Vesta and at the Moon, although at a slower pace, the properties of the Vesta surface material may limit implanted proton-induced hydroxylation.

Thus, detection of $\mathrm{H}$ and $\mathrm{OH} / \mathrm{H}_{2} \mathrm{O}$ on Vesta (or not), and correlations with Vesta surface properties, is key to understand the underlying process(es) and their potential for affecting other Solar System bodies.

\section{Concluding Remarks}

As the Vesta data from Dawn's Framing Camera (FC), Visible and Infrared imaging spectrometer (VIR), and Gamma Ray and Neutron Detector (GRaND) are acquired and analyzed in an integrated manner along with key radio-science information constraining Vesta's interior, an assessment of the composition and history of the only known differentiated silicate proto-planet of our solar system will emerge. The integrated approach for example science themes discussed above is summarized in Table 2. The integration of these diverse data will take some time since each of the mission phases brings increasingly more complete information from each instrument (Russell and Raymond 2011). Some of the science issues outlined above will be resolved quickly, others more slowly, and a plethora of new questions (currently unknown) will be urgently posed. As Vesta is gradually revealed by Dawn, her character and secrets will be sure to both delight and surprise us. 
Table 2 Example Science Themes evaluated by Dawn at Vesta

\begin{tabular}{|c|c|c|c|}
\hline Theme & Scientific questions & Measurements & Instruments \\
\hline \multirow[t]{4}{*}{ Interior of Vesta } & $\begin{array}{l}\text { Are the mantle and lower crust } \\
\text { exposed? }\end{array}$ & $\begin{array}{l}\text { Spectra and color images of } \\
\text { central peaks, walls, floor } \\
\text { and ejecta. }\end{array}$ & $\begin{array}{l}\text { VIR, FC + Gravity } \\
\text { and topography }\end{array}$ \\
\hline & Model I: Layered interior & $\begin{array}{l}\text { Signature of olivine and py- } \\
\text { roxene bearing lithologies in } \\
\text { regular spatial pattern. }\end{array}$ & $\begin{array}{l}\text { VIR, FC + Gravity } \\
\text { and topography }\end{array}$ \\
\hline & $\begin{array}{l}\text { Model II: Interior reaccreted } \\
\text { and reaggregated }\end{array}$ & $\begin{array}{l}\text { Distribution and scale of } \\
\text { materials in random com- } \\
\text { positional variations. }\end{array}$ & $\begin{array}{l}\text { VIR, FC + Gravity } \\
\text { and topography }\end{array}$ \\
\hline & $\begin{array}{l}\text { Is there evidence of diverse } \\
\text { basaltic magmatism across the } \\
\text { surface? }\end{array}$ & $\begin{array}{l}\text { Elemental, mineralogical, } \\
\text { and color maps in spatial } \\
\text { context. }\end{array}$ & $\begin{array}{l}\text { GRAND, FC } \\
\text { and VIR }\end{array}$ \\
\hline \multirow[t]{4}{*}{$\begin{array}{l}\text { Space Weathering } \\
\text { on Vesta }\end{array}$} & $\begin{array}{l}\text { Is space weathering absent } \\
\text { across Vesta's surface? }\end{array}$ & $\begin{array}{l}\text { Elemental, spectral and } \\
\text { color global maps. }\end{array}$ & $\begin{array}{l}\text { GRAND, FC } \\
\text { and VIR }\end{array}$ \\
\hline & $\begin{array}{l}\text { If present, what is the signa- } \\
\text { ture of weathering products on } \\
\text { Vesta? }\end{array}$ & $\begin{array}{l}\text { Spectra of fresh and old } \\
\text { materials (absorption bands } \\
\text { and near-infrared contin- } \\
\text { uum). }\end{array}$ & VIR, FC \\
\hline & $\begin{array}{l}\text { What is the total exposure of } \\
\text { the surface to solar wind? }\end{array}$ & $\begin{array}{l}\mathrm{H} \text { abundance across the sur- } \\
\text { face }\end{array}$ & GRAND \\
\hline & $\begin{array}{l}\text { Is the surface of Vesta ancient } \\
\text { or recently resurfaced? }\end{array}$ & $\begin{array}{l}\text { Morphology; compositional } \\
\text { unit definition; Crater fre- } \\
\text { quency distribution. }\end{array}$ & $\begin{array}{l}\text { FC, VIR, } \\
\text { and GRAND }\end{array}$ \\
\hline \multirow[t]{3}{*}{$\begin{array}{l}\mathrm{H}, \mathrm{HO}, \mathrm{H}_{2} \mathrm{O} \text { and } \\
\text { other Volatiles on Vesta }\end{array}$} & $\begin{array}{l}\text { Does } \mathrm{OH} / \mathrm{H}_{2} \mathrm{O} \text { form on airless } \\
\text { bodies? }\end{array}$ & $\begin{array}{l}\mathrm{OH} \text { and } \mathrm{H}_{2} \mathrm{O} \text { absorption } \\
\text { features }\end{array}$ & VIR \\
\hline & $\begin{array}{l}\text { What other volatiles are } \\
\text { present? }\end{array}$ & $\begin{array}{l}\mathrm{CO}_{2} \text { or other volatile ab- } \\
\text { sorption features }\end{array}$ & VIR, GRAND \\
\hline & $\begin{array}{l}\text { How does the solar wind inter- } \\
\text { act with surface material? }\end{array}$ & $\begin{array}{l}\mathrm{H} \text {, temporal and regional } \\
\text { distribution of } \mathrm{OH} \text { and } \mathrm{H}_{2} \mathrm{O}\end{array}$ & GRAND, VIR \\
\hline
\end{tabular}

Acknowledgements NASA support for this work is greatly appreciated, including NASA contract NNM05AA86C, subcontract \#2090 S JB693. We gratefully acknowledge the kind permission of N. Moskovitz to include some of his V-type asteroid data in this overview. Reviews by C.R. Chapman and an anonymous reviewer were quite helpful and appreciated.

Open Access This article is distributed under the terms of the Creative Commons Attribution Noncommercial License which permits any noncommercial use, distribution, and reproduction in any medium, provided the original author(s) and source are credited.

\section{References}

J.F. Bell, III, N.I. Izenberg, P.G. Lucey, B.E. Clark, C. Peterson, M.J. Gaffey, J. Joseph, B. Carcich, A. Harch, M.E. Bell, J. Warren, P.D. Martin, L.A. McFadden, D. Wellnitz, S. Murchie, M. Winter, J. Veverka, P. Thomas, M.S. Robinson, M. Malin, A. Cheng, Near-IR reflectance spectroscopy of 433 Eros from the NIS instrument on the NEAR mission: I. Low phase angle observations. Icarus 155, 119-144 (2002)

R.P. Binzel, S. Xu, Chips off of asteroid 4 Vesta: evidence for the parent body of basaltic achondrite meteorites. Science 260, 186-191 (1993) 
R.P. Binzel, S.J. Bus, T.H. Burbine, J.M. Sunshine, Spectral properties of near-Earth asteroids: evidence for sources of ordinary chondrite meteorites. Science 273, 946-948 (1996)

R.P. Binzel, M.J. Gaffey, P.C. Thomas, B.H. Zellner, A.D. Storrs, E.N. Wells, Geologic mapping of Vesta from 1994 Hubble Space Telescope images. Icarus 128, 95-103 (1997)

R.P. Binzel, A.S. Rivkin, S.J. Bus, J.M. Sunshine, T.H. Burbine, MUSES-C target asteroid (25143) 1998 SF36: a reddened ordinary chondrite. Meteorit. Planet. Sci. 36, 1167-1172 (2001)

R.P. Binzel, A.S. Rivkin, J.S. Stuart, A.W. Harris, S.J. Bus, T.H. Burbine, Observed spectral properties of near-Earth objects: results for population distribution, source regions, and space weathering processes. Icarus 170, 259-294 (2004)

W.F. Bottke Jr., D. Vokrouhlický, D.P. Rubincam, D. Nesvorný, The Yarkovsky and Yorp effects: implications for asteroid dynamics. Annu. Rev. Earth Planet. Sci. 34, 157-191 (2006)

R.G. Burns, Mineralogical Applications of Crystal Field Theory, 2nd edn., p 551. Cambridge University Press, New York (1993)

B. Carry, P. Vernazza, C. Dumas, M. Fulchignoni, Icarus 205, 473-482 (2010)

W. Cassidy, B. Hapke, Effects of darkening processes on surfaces on surfaces of airless bodies. Icarus 25, 371-383 (1975)

C.R. Chapman, S-type asteroids, ordinary chondrites, and space weathering: the evidence from Galileo's fly-bys of Gaspra and Ida. Meteorit. Planet. Sci. 31, 699-725 (1996)

C.R. Chapman, Space weathering on asteroid surfaces. Annu. Rev. Earth Planet. Sci. 32, 539-567 (2004)

A.F. Cheng, Near Earth asteroid rendezvous: mission summary, in Asteroids III, ed. by W. Bottke et al. (University of Arizona Press, Tucson, 2002), pp. 351-366

B.E. Clark, B. Hapke, C.M. Pieters, D. Britt, Asteroid space weathering and regolith evolution, in Asteroids III, ed. by W. Bottke et al. (University of Arizona Press, Tucson, 2002), pp. 585-599

R.N. Clark, Detection of adsorbed water and hydroxyl on the Moon. Science 326, 562-564 (2009)

A. Colaprete, K. Ennico, D. Wooden, M. Shirle, J. Heldmann, W. Marshall, L. Sollitt, E. Asphaug, D. Korycansky, P. Schultz, B. Hermalyn, K. Galal, G.D. Bart, D. Goldsteie, D. Summy, in Water and More: an Overview of LCROSS Impact Results, 41st LPSC, vol. 1533 (2010a), p. 2335

A. Colaprete, P. Schultz, J. Heldmann, D. Wooden, M. Shirley, K. Ennico, B. Hermalyn, W. Marshall, A. Ricco, R.C. Elphic, D. Goldstein, D. Summy, G.D. Bart, E. Asphaug, D. Korycansky, D. Landis, L. Sollitt, Detection of water in the LCROSS ejecta plume. Science 330, 463-468 (2010b)

A. Coradini, D. Turrini, C. Federico, G. Magni, Vesta and Ceres: crossing the history of the Solar system. Space Sci. Rev. (2011, in press)

M.C. De Sanctis, A. Coradini, E. Ammannito, G. Filacchione, M.T. Capria, S. Fonte, G. Magni, A. Barbis, A. Bini, M. Dami, I. Ficai-Veltroni, G. Preti, VIR Team, The VIR spectrometer. Space Sci. Rev. (2011a). doi:10.1007/s11214-010-9668-5

M.C. De Sanctis, A. Migliorini, F.L. Jasmin, D. Lazzaro, G. Filacchione, S. Marchi, E. Ammannito, M.T. Capria, Spectral and mineralogical characterization of inner main belt V-type asteroids. Astron. Astrophys. (2011b, in press)

R. Duffard, D. Lazzaro, J. Licandro, M.C. De Sanctis, M.T. Capria, J.M. Carvano, Icarus 171, 120 (2004)

V.R. Eke, L.F.A. Teodoro, R.C. Elphic, The spatial distribution of polar hydrogen deposits on the Moon. Icarus 200(1), 12-18 (2009)

W.C. Feldman, S. Maurice, A.B. Binder, B.L. Barrowclough, R.C. Elphic, D.J. Lawrence, Fluxes of fast and epithermal neutrons from lunar prospector: evidence for water ice at the lunar poles. Science 281, 1496 (1998). doi:10.1126/science.281.5382.1496

W.C. Feldman, D.J. Lawrence, R.C. Elphic, B.L. Barraclough, S. Maurice, L. Genetay, A.B. Binder, Polar hydrogen deposits on the Moon. J. Geophys. Res. 105(E2), 4175-4196 (2000)

W.C. Feldman, S. Maurice, D.J. Lawrence, R.C. Little, S.L. Lawson, O. Gasnault, R.C. Wiens, B.L. Barraclough, R.C. Elphic, T.H. Prettyman, J.T. Steinberg, A.B. Binder, Evidence for water ice near the lunar poles. J. Geophys. Res. 106(E10), 23231 (2001)

M.J. Gaffey, T.H. Burbine, R.P. Binzel, Asteroid spectroscopy: progress and perspectives. Meteorit. 28, 161187 (1993)

M.J. Gaffey, Surface lithologic heterogeneity of Asteroid 4 Vesta. Icarus 127, 130-157 (1997)

M.J. Gaffey, Space weathering and the interpretation of asteroid reflectance spectra. Icarus 209, 564-574 (2010)

B. Hapke, Space weathering from Mercury to the asteroid belt. J. Geophys. Res. 106, 10039-10073 (2001)

S. Hasegawa, K. Murakawa, M. Ishiguro, H. Nonaka, N. Takato, C.J. Davis, M. Ueno, T. Hiroi, Evidence of hydrated and/or hydroxylated minerals on the surface of Asteroid 4 Vesta. Geophys. Res. Lett. 30(21), 2123 (2003). doi:10.1029/2003GL018627

E.K. Hauri, T. Weinreich, A.E. Saal, M.C. Rutherford, J.A. Van Orman, High pre-eruptive water contents preserved in lunar melt inclusions. Science 333(6039), 213-215 (2011). doi:10.1126/science.1204626 
T. Hiroi, C.M. Pieters, H. Takeda, Grain size of the surface regolith of Asteroid 4 Vesta estimated from its reflectance spectrum in comparison with HED meteorites. Meteorit. 29, 394-396 (1994)

T. Hiroi, R.P. Binzel, J.M. Sunshine, C.M. Pieters, H. Takeda, Grain sizes and mineral compositions of surface regoliths of Vesta-like asteroids. Icarus 115, 374-386 (1995)

T. Hiroi, C.M. Pieters, Origin of Vestoids suggested from the space weathering trend in the visible reflectance spectra of HED meteorites and lunar soils. Antarct. Meteor. Res. 11, 165-172 (1998)

T. Hiroi, S. Sasaki, Importance of space weathering simulation products in compositional modeling of asteroids: 349 Dembowska and 446 Aeternitas as examples. Meteorit. Planet. Sci. 36, 1587-1596 (2001)

T. Hiroi, M. Abe, K. Kitazato, S. Abe, B.E. Clark, S. Sasaki, M. Ishiguro, O.S. Barnouin-Jha, Developing space weathering on the asteroid 25143 Itokawa. Nature 443, 56-58 (2006)

M. Ishiguro, T. Hiroi, D.J. Tholen, S. Sasaki, Y. Ueda, T. Nimura, M. Abe, B.E. Clark, A. Yamamoto, F. Yoshida, R. Nakamura, N. Hirata, H. Miyamoto, Y. Yokota, T. Hashimoto, T. Kubota, A.M. Nakamura, R.W. Gaskell, J. Saito, Global mapping of the degree of space weathering on asteroid 25143 Itokawa by Hayabusa/AMICA observations. Meteorit. Planet. Sci. 42, 1791-1800 (2007)

R. Jaumann, G. Neukum, T. Behnke, T.C. Duxbury, J. Flohrer, S. van Gasselt, B. Giese, K. Gwinner, E. Hauber, H. Hoffmann, U. Köhler, K.-D. Matz, T.B. McCord, V. Mertens, J. Oberst, R. Pischel, D. Reiss, T. Roatsch, P. Saiger, F. Scholten, G. Schwarz, K. Stephan, M. Wählisch, The high resolution stereo camera (HRSC) experiment on Mars Express: instrument aspects and experiment conduct from interplanetary cruise through the nominal mission. Planet. Space Sci. 55, 928-952 (2007)

M. Jutzi, E. Asphaug, Mega-ejecta on asteroid Vesta. Geophys. Res. Lett. 38, L01102 (2011). doi:10.1029/ 2010GL045517

L.P. Keller, D.S. McKay, Discovery of vapor deposits in the lunar regolith. Science 261, 1305-1307 (1993)

L.P. Keller, D.S. McKay, The nature and origin of rims on lunar soil grains. Geochim. Cosmochim. Acta 61, 2331-2341 (1997)

D.J. Lawrence, W.C. Feldman, R.C. Elphic, J.J. Hagerty, S. Maurice, G.W. McKinney, T.H. Prettyman, Improved modeling of Lunar Prospector neutron spectrometer data: implications for hydrogen deposits at the lunar poles. J. Geophys. Res. 111, E08001 (2006). doi:10.1029/2005JE002637

D.J. Lawrence, R.C. Elphic, W.C. Feldman, H.O. Funsten, T.H. Prettyman, Astrobiology 10(2), 183-200 (2010). doi:10.1089/ast.2009.0401

J.-Y. Li, L.A. McFadden, P.C. Thomas, M.J. Mutchler, J.W. Parker, E.F. Young, C.T. Russell, M.V. Sykes, B.E. Schmidt, Photometric mapping of Asteroid (4) Vesta's southern hemisphere with Hubble Space Telescope. Icarus 208, 238-251 (2010)

S. Marchi, R. Brunetto, S. Magrin, M. Lazzarin, D. Gandolfi, Astron. Astrophys. 443, 769 (2005)

S. Marchi, M.C. De Sanctis, M. Lazzarin, S. Magrin, Astrophys. J. Lett. 721, L172 (2010)

T.H. McCoy, T.H. Burbine, L.A. McFadden, R.D. Starr, M.J. Gaffey, L.R. Nittler, L.G. Evans, N. Izenberg, P.G. Lucey, J.I. Trombka, J.F. Bell III, B.E. Clark, P.E. Clark, S.W. Squyres, C.R. Chapman, W.V. Boynton, J. Veverka, The composition of 433 Eros: a mineral-chemical synthesis. Meteorit. Planet. Sci. 36, 1661-1672 (2001)

T.B. McCord, J.B. Adams, T.V. Johnson, Asteroid Vesta: spectral reflectivity and compositional implications. Science 168, 1445-1447 (1970)

T.B. McCord, L.A. Taylor, T.M. Orlando, R.N. Clark, C.M. Pieters, J.-Ph. Combe, G.Y. Kramer, J.M. Sunshine, M.D. Dyar, C.A. Hibbitts, Interpretations of $\mathrm{OH} / \mathrm{HOH}$ IR Absorptions on the Moon from Chandrayaan-1 Moon Mineralogy Mapper (Am. Geophys. Union, Washington, 2009). Fall Meeting 2009, abstract \#P34A-04

T.B. McCord, L.A. Taylor, T.M. Orlando, C.M. Pieters, J.-Ph. Combe, G. Kramer, J.M. Sunshine, J.W. Head, J.F. Mustard, Origin of OH/Water on the Lunar surface detected by the Moon Mineralogy Mapper. LPSC \#1860 (2010a)

T.B. McCord, J.-Ph. Combe, L.A. Taylor, G.Y. Kramer, C.M. Pieters, J.M. Sunshine, J. Boardman, Nature and potential causes for 3- $\mu \mathrm{m}$ absorptions in the lunar reflectance spectrum due to $\mathrm{OH} / \mathrm{H}_{2} \mathrm{O}$ as discovered by the Moon Mineralogy Mapper on the Chandrayaan-1 Spacecraft. European Geoscience Union General Assembly, Vienna, Austria, 2-7 May 2010, EGU2010-6137 (2010b)

T.B. McCord, J.-Ph. Combe, L.A. Taylor, G.Y. Kramer, C.M. Pieters, J.M. Sunshine, J. Boardman, Sources and physical processes responsible for $\mathrm{OH} / \mathrm{H}_{2} \mathrm{O}$ in the lunar soil as revealed by the Moon Mineralogy Mapper (M $\left.{ }^{3}\right)$. J. Geophys. Res. (2010c). doi:10.1029/2010JE003711

H.Y. McSween, D.W. Mittlefehldt, A.W. Beck, R.G. Mayne, T.J. McCoy, HED meteorites and their relationship to the geology of Vesta and the Dawn mission. Space Sci. Rev. (2010). doi:10.1007/ s11214-010-9637-z

I. Mitrofanov et al., LEND experiment onboard LRO: testing local areas with high concentrations of hydrogen at the lunar poles. LPSC, 2250 (2010a)

I.G. Mitrofanov, A. Bartels, Y.I. Bobrovnitsky, W. Boynton, G. Chin, H. Enos, L. Evans, S. Floyd, J. Garvin, D.V. Golovin, A.S. Grebennikov, K. Harshman, L.L. Kazakov, J. Keller, A.A. Konovalov, A.S. Kozyrev, 
A.R. Krylov, M.L. Litvak, A.V. Malakhov, T. McClanahan, G.M. Milikh, M.I. Mokrousov, S. Ponomareva, R.Z. Sagdeev, A.B. Sanin, V.V. Shevchenko, V.N. Shvetsov, R. Starr, G.N. Timoshenko, T.M. Tomilina, V.I. Tretyakov, J. Trombka, V.S. Troshin, V.N. Uvarov, A.B. Varennikov, A.A. Vostrukhin, Lunar Exploration Neutron Detector for the NASA Lunar Reconnaissance Orbiter. Space Sci. Rev. 150, 183-207 (2010b). doi:10.1007/s11214-009-9608-4

I.G. Mitrofanov, A.B. Sanin, W. Boynton, G. Chin, J. Garvin, D.V. Golovin, L.G. Evans, K. Harshman, A.S. Kozyrev, M.L. Litvak, A.V. Malakhov, E. Mazarico, T. McClanahan, G. Milikh, M. Mokrousov, G. Nandikotkur, G.A. Neumann, I. Nuzhdin, R. Sagdeev, V. Shevchenko, V. Shvetsov, D.E. Smith, R. Starr, V.I. Tretyakov, J. Trombka, D. Usikov, A. Varenikov, A. Vostrukhin, M.T. Zuber, Hydrogen mapping of the Lunar South Pole using the LRO neutron detector experiment LEND. Science 330, 483-486 (2010c)

M. Miyamoto, H. Takeda, Evidence for excavation of deep crustal material of a Vesta-like body from Ca compositional gradients in pyroxene. Earth Planet. Sci. Lett. 122, 343 (1994)

L.V. Moroz, A.V. Fisenko, L.F. Semjonova, C.M. Pieters, N.N. Korotaeva, Optical effects of regolith processes on $\mathrm{S}$-asteroids as simulated by laser shots on ordinary chondrite and other mafic materials. Icarus 122, 366-382 (1996)

N.A. Moskovitz, M. Willman, T.H. Burbine, R.P. Binzel, S.J. Bus, A spectroscopic comparison of HED meteorites and V-type asteroids in the inner main belt. Icarus 208, 773-788 (2010)

J.W. Nettles, M. Staid, S. Besse, J. Boardman, R.N. Clark, D. Dhingra, P. Isaacson, R. Klima, G. Kramer, C.M. Pieters, L.A. Taylor, Optical maturity variation in lunar spectra as measured by Moon Mineralogy Mapper data. J. Geophys. Res. (2010). doi:10.1029/2010JE003748

D. Nesvorný, D. Vokrouhlický, New candidates for recent asteroid breakups. Astron. J. 132, 1950-1958 (2006)

S.K. Noble, C.M. Pieters, L.A. Taylor, R.V. Morris, C.C. Allen, D.S. McKay, L.P. Keller, Optical properties of the finest fraction of lunar soils: implications for space weathering environments. Meteorit. Planet. Sci. 36, 31-42 (2001)

S.K. Noble, C.M. Pieters, L.P. Keller, An experimental approach to understanding the optical effects of space weathering. Icarus 192, 629-642 (2007). doi:10.1016/j.icarus.2007.07.021

S.K. Noble, L.P. Keller, C.M. Pieters, Evidence of space weathering in regolith breccias II: asteroidal regolith breccias. Meteorit. Planet. Sci. 45(12), 2007-2015 (2010)

C.M. Pieters, R.P. Binzel, Young Vesta (regolith)? Lunar Planet. Sci. Conf. 25, 1083-1084 (1994)

C.M. Pieters, L.A. McFadden, Meteorite and asteroid reflectance spectroscopy: clues to early Solar system processes. Annu. Rev. Earth Planet. Sci. 22, 457-497 (1994)

C.M. Pieters, L.A. Taylor, Systematic global mixing and melting in lunar soil evolution. Geophys. Res. Lett. 30, (20), 2048 (2003). doi:10.1029/2003GL018212

C.M. Pieters, L.A. Taylor, S.K. Noble, L.P. Keller, B. Hapke, R.V. Morris, C.C. Allen, D.S. McKay, S. Wentworth, Space weathering on airless bodies: resolving a mystery with lunar samples. Meteorit. Planet. Sci. 35, 1101-1107 (2000)

C.M. Pieters, R.P. Binzel, D.D. Bogard, T. Hiroi, D.W. Mittlefehldt, L.E. Nyquist, A.S. Rivkin, H. Takeda, Asteroid-meteorite links: the Vesta conundrum(s), in Asteroids, Comets, Meteors, 229th Symposium of the International Astronomical Union (2006), pp. 273-288. doi:10.1017/S1743921305006794

C.M. Pieters, J.N. Goswami, R.N. Clark, M. Annadurai, J. Boardman, B. Buratti, J.P. Combe, M.D. Dyar, R. Green, J.W. Head, C. Hibbitts, M. Hicks, P. Isaacson, R. Klima, G. Kramer, S. Kumar, E. Livo, S. Lundeen, E. Malaret, T. McCord, J. Mustard, J. Nettles, N. Petro, C. Runyon, M. Staid, J. Sunshine, L.A. Taylor, S. Tompkins, P. Varanasi, Character and spatial distribution of $\mathrm{OH} / \mathrm{H}_{2} \mathrm{O}$ on the surface of the Moon seen by M3 on Chandrayaan-1. Science 326, 568-572 (2009)

T.H. Prettyman, W.C. Feldman, H.Y. McSween Jr., R.D. Dingler, D.C. Enemark, D.E. Patrick, S.A. Storms, J.S. Hendricks, J.P. Morgenthaler, K.M. Pitman, R.C. Reedy, Dawn's Gamma ray and neutron detector. Space Sci. Rev. (2011, in press)

C. Raymond et al., Dawn Topographic Investigations (2011, this issue)

V. Reddy, M.J. Gaffey, M.S. Kelley, A. Nathues, J.-Y. Li, R. Yarbrough, Compositional heterogeneity of Asteroid 4 Vesta's southern hemisphere: implications for the Dawn mission. Icarus 210, 693-706 (2010)

V. Reddy, A. Nathues, M.J. Gaffey, First fragment of Asteroid 4 Vesta's mantle detected. Icarus 212, 175-179 (2011)

A.S. Rivkin, L.A. McFadden, R.P. Binzel, M. Sykes, Rotationally-resolved spectroscopy of Vesta I: 2-4 $\mu \mathrm{m}$ region. Icarus 180, 464-472 (2006)

C.T. Russell, C.A. Raymond, The Dawn discovery mission to Vesta and Ceres. Space Sci. Rev. (2011, in press)

C.T. Russell et al., Dawn mission to Vesta and Ceres: symbiosis between terrestrial observations and robotic exploration. Earth Moon Planets 101, 65-91 (2007) 
A.E. Saal, E.H. Hauri, M.L. Cascio, J.A. van Orman, M.C. Rutherford, R.F. Cooper, Volatile content of lunar volcanic glasses and the presence of water in the Moon's interior. Nature 454, 192-195 (2008). doi:10.1038/nature07047

S. Sasaki, K. Nakamura, Y. Hamabe, E. Kurahashi, T. Hiroi, Production of iron nanoparticles by laser irradiation in a simulation of lunar-like space weathering. Nature 410, 555-557 (2001)

P.H. Schultz, B. Hermalyn, A. Colaprete, K. Ennico, M. Shirley, W.S. Marshall, The LCROSS cratering experiment. Science 330, 468-472 (2010)

H. Sierks, H.U. Keller, R. Jaumann, H. Michalik, F. Bubenhagen, I. Büttner, U. Carsenty, U. Christensen, R. Enge, F. Fiethe, P. Gutiérrez Marqués, H. Hartwig, H. Krüger, W. Kühne, T. Maue, S. Mottola, A. Nathues, K.-U. Reiche, M.L. Richards, T. Roatsch, S.E. Schröder, I. Szemerey, M. Tschentscher, The Dawn framing camera (2010, this issue). doi:10.1007/s11214-011-9745-4

G. Strazzulla, E. Dotto, R. Binzel, R. Brunetto, M.A. Barucci, A. Blanco, V. Orofino, Spectral alteration of the Meteorite Epinal (H5) induced by heavy ion irradiation: a simulation of space weathering effects on near-Earth asteroids. Icarus 174, 31-35 (2005)

J.M. Sunshine, T.L. Farnham, L.M. Feaga, O. Groussin, F. Merlin, R.E. Milliken, M.F. A'Hearn, Temporal and spatial variability of lunar hydration as observed by the Deep Impact spacecraft. Science 326, 565568 (2009)

H. Takeda, A layered-crust model of a howardite parent body. Icarus 40, 455-470 (1979)

H. Takeda, Mineralogical records of early planetary processes on the howardite, eucrite, diogenite parent body with reference to Vesta. Meteorit. Planet. Sci. 32, 841 (1997)

L.A. Taylor, H.K. Mao, P.M. Bell, "Rust" in the Apollo 16 rocks. Proc. Lunar Planet. Sci. Conf. 4, 829 (1973)

L.A. Taylor, H.K. Mao, P.M. Bell, Identification of the hydrated iron oxide mineral akaganéite in Apollo 16 lunar rocks. Geology 2, 429-432 (1974). doi:10.1130/0091-7613

L.A. Taylor, C.M. Pieters, R.V. Morris, L.P. Keller, D.S. McKay, Lunar mare soils: space weathering and the major effects of surface-correlated nanophase Fe. J. Geophys. Res. 106, 27985-28000 (2001)

P.C. Thomas, R.R. Binzel, M.J. Gaffey, B.H. Zellner, A.D. Storrs, E. Wells, Vesta: spin pole, size, and shape from HST images. Icarus 128, 88-94 (1997)

A.H. Treiman, A. Lanzirotti, D. Xirouchakis, Ancient water on Asteroid 4 Vesta: evidence from a quartz veinlet in the Serra de Magé eucrite meteorite. Earth Planet. Sci. Lett. 219, 189-199 (2004)

M. Yamada, S. Sasaki, H. Nagahara, A. Fujiwara, S. Hasegawa, H. Yano, T. Hiroi, H. Ohashi, H. Ohtake, Simulation of space weathering of planet-forming materials: nanosecond pulse laser irradiation and proton implantation on olivine and pyroxene samples. Earth Planets Space 51, 1255-1265 (1999)

P. Vernazza, T. Mothe-Diniz, M.A. Barucci, M. Birlan, J.M. Carvano, G. Strazzulla, M. Fulchignoni, A. Migliorini, Analysis of near-IR spectra of 1 Ceres and 4 Vesta, targets of the Dawn mission. Astron. Astrophys. 436, 1113-1121 (2005)

P. Vernazza, R. Brunetto, G. Strazzulla, M. Fulchignoni, P. Rochette, N. Meyer-Vernet, I. Zouganelis, Asteroid colors: a novel tool for magnetic field detection? The case of Vesta. Astron. Astrophys. 451, 43-46 (2006)

P. Vernazza, R. Binzel, M. Birlan, M. Fulchignoni, A. Rossi, Solar wind as the origin of rapid reddening of asteroid surfaces. Nature 458, 993-995 (2009)

J. Wasson, C.R. Chapman, K. Grogam, S.F. Dermott, Possible formation of the Vesta-family asteroids and the main IRAS dust band by an oblique impact on Vesta. Lunar Planet. Sci. Conf. 27, 1387-1388 (1996)

J. Wisdom, Meteorites may follow a chaotic route to Earth. Nature 315, 731 (1985)

E.J. Zeller, L.B. Ronca, P.W. Levy, Proton-induced hydroxyl formation on the lunar surface. J. Geophys. Res. 71, 4855-4860 (1966)

A.P. Zent, A.I. Ichimura, T.B. McCord, L.A. Taylor, Production of $\mathrm{OH} / \mathrm{H}_{2} \mathrm{O}$ in lunar samples via proton bombardment. LPSC 41, 2665 (2010)

M.T. Zuber, H.Y. McSween Jr., R.P. Binzel, L.T. Elkins-Tanton, A.S. Konopliv, C.M. Pieters, D.E. Smith, Origin, Internal structure and evolution of 4 Vesta. Space Sci. Rev. (2011, this issue). doi:10.1007/ s11214-011-9806-8 